\title{
Cuidado de enfermagem em urgência/emergência às pessoas que tentam suicídio*
}

\author{
Mayara Cristine Fontão ${ }^{1}$ \\ (D) https://orcid.org/0000-0002-0798-1257 \\ Jeferson Rodrigues ${ }^{2}$ \\ iD https://orcid.org/0000-0002-8612-9088 \\ Monica Mota Lino 2 \\ (D) https://orcid.org/0000-0003-0828-7969 \\ Murielk Motta Lino ${ }^{3}$ \\ (D) https://orcid.org/0000-0002-9286-373X
}

Objetivo: trata-se de uma revisão integrativa que teve como objetivo caracterizar o cuidado de Enfermagem em urgência/ emergência às pessoas que tentam suicídio. Método: a busca contemplou as bases de dados CINAHL $^{\circledR}$, SCOPUS ${ }^{\circledR}$, SciElo ${ }^{\circledR}$, $\operatorname{LILACS}^{\circledR}, \operatorname{MEDLINE}^{\circledR}$ e Google Acadêmico ${ }^{\circledR}$ no período de 2011 a 2018. Resultados: a partir do universo de 5900 estudos, foram selecionados 16 artigos para o corpus de análise aprofundada. Emergiram três eixos centrais: caracterização do tema suicídio, trabalho e percepção da Enfermagem no cuidado e aspectos que envolvem o cuidado na área de urgência/emergência. Conclusão: os resultados dos estudos apontaram a importância de aprofundamento do tema, principalmente quanto ao estigma social que o profissional de Enfermagem apresenta ao prestar cuidado à pessoa em tentativa de suicídio, para trazer melhorias ao cuidado.

Descritores: Cuidados de Enfermagem; Tentativa de Suicídio; Serviços de Emergência Psiquiátrica; Saúde Mental; Estigma Social.

\section{Como citar este artigo}

Fontão MC, Rodrigues J, Lino MM, Lino MM. Nursing care in Urgency/Urgency/Emergency to people who attempt suicide. SMAD, Rev Eletrônica Saúde Mental Álcool Drog. 2020;16(4):122-132. doi: https://dx.doi.org/10.11606/issn.1806-6976.smad.2020.152045 


\title{
Nursing care in Urgency/Urgency/Emergency to people who attempt suicide
}

\begin{abstract}
Objective: this is an integrative review that aimed to characterize emergency/emergency nursing care for people who attempt suicide. Method: the search contemplated the CINAHL ${ }^{\circledR}, \mathrm{SCOPUS}^{\circledR}$, SciElo ${ }^{\circledR}$, LILACS $^{\circledR}$, MEDLINE $^{\circledR}$ and Google Academic ${ }^{\circledR}$ databases in the period from 2011 to 2018. Results: From the universe of 5900 studies, 16 articles were selected for the corpus of in-depth analysis. Three central axes emerged: characterization of the suicide theme, work and perception of nursing in care and aspects involving care in the area of urgency/emergency. Conclusion: the results of the studies pointed out the importance of deepening the theme, especially regarding the social stigma that the Nursing professional presents when caring for a person in a suicide attempt, in order to bring improvements to care.
\end{abstract}

Descriptors: Nursing Care; Suicide Attempted; Emergency Services, Psychiatric; Mental Health; Social Stigma.

\section{Cuidados de enfermería en emergencia a las personas que intentan suicidarse}

Objetivo: se trata de una revisión integradora que tuvo como objetivo caracterizar elcuidado de enfermería en la urgencia médica a las personas que intentan suicidarse. Metodo: la búsqueda incluyó las bases de datos CINAHL, Scopus ${ }^{\circledR}, \mathrm{SciElo}^{\circledR}, \mathrm{LILACS}^{\circledR}$, Medline ${ }^{\circledR}$ y Google Acadêmico ${ }^{\circledR}$ en el período de 2011 a 2018. Resultados: a partir de 5900estudios, se seleccionaron 16 para análisis. Emergido 03 temas centrales: la caracterización del tema del suicidio, el trabajo y la percepción de los cuidados de enfermería y los aspectos relacionados con el cuidado en el área de urgencias médicas. Conclusión: los resultados mostraron la importancia de profundizar el tema, sobre todo debido al estigma social presenteen la prestación decuidados, para introducir mejoras en la atención.

Descriptores: Atención de Enfermería; Intento de Suicidio; Servicios de Urgencia Psiquiátrica; Salud Mental; Estigma Social. 


\section{Introdução}

O suicídio envolve diversos fatores, pois é uma situação complexa, representa um problema de saúde pública e significa todo ato executado pela pessoa que possui a intenção de morrer, utilizando-se de um método no qual acredita que será capaz de terminar com a própria vida, ou seja, é a morte intencional autoinflingida. Portanto, é necessário uma compreensão da situação singular a qual essa pessoa está envolvida(1).

Autores apontaram que comportamentos autolesivos podem ser categorizados como: 1) suicídio completo; 2) tentativa de suicídio; 3) atos preparatórios para o comportamento suicida; 4) ideação suicida; 5) comportamento autoagressivo sem intenção de morrer; 6) automutilação não intencional e 7) automutilação com intenção suicida desconhecida. Toda ameaça apresentada por uma pessoa em situação de vulnerabilidade para o suicídio deve ser levada em consideração, mesmo quando aparenta ser falsa ou de caráter manipulador. A repetição de tentativas de suicídio é um indicador de risco para a consumação do ato(2-3).

A emergência de um hospital geral é considerada uma porta de entrada terciária do Sistema Único de Saúde (SUS). Essa área de urgências é considerada prioritária, pois atende aos agravos de saúde que necessitem de cuidados imediatos. A Política Nacional de Atenção às Urgências tem como objetivo garantir a universalidade, equidade e integralidade no atendimento às urgências e, dentre essas, tem-se a tentativa de suicídio(3).

Sabe-se que $50 \%$ das pessoas que morreram por suicídio tinham, ao menos, uma tentativa prévia e que de 15 a $25 \%$ realizaram uma nova tentativa em menos de um ano. O Brasil encontra-se entre os dez países que registram os maiores números absolutos de suicídios. $\mathrm{E}$ tal ato situa-se como uma das três maiores causas de morte na faixa etária entre 15 e 35 anos e representa a sexta causa de incapacitação dos indivíduos entre 15 e $44 \operatorname{anos}^{(1)}$.

Estima-se que o número de tentativas de suicídio seja de dez a 20 vezes mais frequentes que o suicídio em si. Os transtornos mentais encontram-se presentes na maioria dos casos de suicídio, principalmente; depressão, transtorno do humor e uso abusivo de substâncias psicoativas, que costumam agravar esse comportamento automutilante, quando associados (4).

A abordagem à pessoa com transtorno mental em situação de emergência e, em especial, que tentou suicídio, deve ser realizada com disposição, segurança, prontidão e qualidade, já que esse comportamento é fator determinante na aceitação e adesão pela pessoa ao cuidado(2).
A maioria dos casos de autoagressão é atendida em algum tipo de serviço de saúde, principalmente na emergência, antes de ocorrer uma tentativa fatal de suicídio. Esse primeiro contato é uma oportunidade ímpar para que os profissionais de saúde identifiquem o potencial nível de risco e possam intervir para minimizá-lo. A relação terapêutica é uma importante ferramenta para aumentar esta adesão e obter resultados satisfatórios. Estabelecer um bom relacionamento pode ter impacto significativo na percepção do paciente sobre a qualidade do cuidado oferecido e na prevenção de novas tentativas ${ }^{(3)}$.

Objetivou-se, por este estudo, tendo em vista esse contexto, caracterizar o cuidado de Enfermagem em urgência/emergência às pessoas que tentam suicídio a partir das produções científicas brasileiras.

\section{Método}

Trata-se de uma Revisão Integrativa da Literatura (RIL) dada sua característica que permite ampla abordagem da literatura investigada(5). Adotou-se um protocolo de revisão com validação externa por dois pareceristas: um ad-hoc no método e outro ad-hoc no conteúdo. A RIL foi desenvolvida em

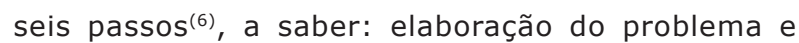
pergunta de pesquisa; busca nas bases de dados científicas; realização da coleta dos dados e registro das informações extraídas dos textos selecionados; análise do conteúdo(7); discussão dos resultados a partir de literatura pertinente e, por fim, apresentação das conclusões.

Foram adotadas as seguintes bases de dados: CINAHL ${ }^{\circledR}$, SCOPUS $^{\circledR}$, SCiElo $^{\circledR}$, LILACS $^{\circledR}$, MEDLINE $^{\circledR}$ e Google Acadêmico ${ }^{\circledR}$, via Portal de Periódicos CAPES. A busca dos estudos ocorreu de modo combinado dos seguintes termos, de dois em dois: "Enfermagem" $O R$ "cuidados de Enfermagem"; "intoxicação exógena" OR "suicídio"; "hospital", "urgência" OR "emergência", publicados no período de 2011 a 2018, em português. Excluíram-se toda produção duplicada, editoriais, teses, dissertações, monografias, inquéritos e textos cuja abordagem metodológica não estivesse clara ou não atendesse ao escopo deste estudo.

Para a organização dos dados utilizou-se o software gerenciador de referências EndNote $W e b ®$ obtendo-se informações referentes ao ano de publicação do estudo, título, objetivos e resultados. A análise do conteúdo foi aplicada a partir da decomposição textual ou de um discurso a partir de unidades de análise para uma categorização e reconstrução de significados que permitiram identificar e interpretar a realidade dos cuidados de Enfermagem às pessoas que tentam suicídio e seus desdobramentos ${ }^{(7)}$. 


\section{Resultados}

Constituiu-se o universo da pesquisa um total de 5.900 estudos. A partir da aplicação dos critérios de exclusão, foram selecionados 16 estudos para a análise aprofundada do conteúdo. Tal detalhamento é apresentado na tabela 1 .

Os estudos utilizados na revisão integrativa foram distribuídos na Figura 1, conforme ano de publicação e título.

Emergiram três eixos centrais: contextualização do tema suicídio no âmbito da urgência e emergência; trabalho da Enfermagem de urgência/emergência frente às pessoas que tentam suicídio; particularidades e necessidades no cuidado ao paciente suicida.

Tabela 1 - Distribuição segundo bases de dados e critérios de inclusão/exclusão. Florianópolis, SC, Brasil, 2019

\begin{tabular}{lccc}
\hline Base de dados & $\begin{array}{c}\text { Critérios de } \\
\text { Inclusão }\end{array}$ & $\begin{array}{c}\text { Critérios de } \\
\text { Exclusão }\end{array}$ & Total \\
\hline CINAHL $^{\oplus}$ & 76 & 76 & - \\
SCOPUS $^{\circledast}$ & 55 & 55 & - \\
SciELO $^{\circledR}$ & 1899 & 1896 & 3 \\
MEDLINE $^{\circledR}$ & 386 & 386 & - \\
LILACS $^{\circledR}$ & 605 & 602 & 3 \\
Google Acadêmico $^{\circledast}$ & 2879 & 2869 & 10 \\
\hline
\end{tabular}

\begin{tabular}{|c|c|l|}
\hline Estudos & Ano & \multicolumn{1}{|c|}{ Título } \\
\hline Estudo 1 & 2017 & $\begin{array}{l}\text { Lesão autoprovocada em todos os ciclos da vida: perfil das vítimas em serviços de urgência e emergência de } \\
\text { capitais do Brasil }\end{array}$ \\
\hline Estudo 2 & 2016 & $\begin{array}{l}\text { Formas utilizadas para tentativa de suicídio e características sociodemográficas de pacientes atendidos no serviço } \\
\text { de emergência de um hospital de ensino }\end{array}$ \\
\hline Estudo 3 & 2015 & Comportamento suicida: percepções e práticas de cuidado \\
\hline Estudo 4 & 2014 & Administração de medicação de uso quando necessário e o cuidado de enfermagem psiquiátrica \\
\hline Estudo 5 & 2014 & Homens envenenados como sujeitos do cuidar e dos cuidados de enfermagem \\
\hline Estudo 6 & 2014 & Cuidado no hospital psiquiátrico sob a ótica da equipe de enfermagem \\
\hline Estudo 7 & 2013 & Impacto do estigma da loucura sobre a atenção de enfermagem ao paciente psiquiátrico em situação de emergência \\
\hline Estudo 8 & 2013 & $\begin{array}{l}\text { Indicadores clínicos do diagnóstico de enfermagem processos familiares disfuncionais em alcoolistas: revisão } \\
\text { integrativa }\end{array}$ \\
\hline Estudo 9 & 2013 & Comunicação entre equipe de enfermagem e pacientes com transtorno mental em um serviço de emergência \\
\hline Estudo 10 & 2013 & Saúde do Homem: internações hospitalares por intoxicação registradas em um centro de assistência toxicológica \\
\hline Estudo 11 & 2013 & Tentativa de suicídio de mulheres: dados de um centro de assistência toxicológica do Paraná \\
\hline Estudo 12 & 2012 & Cuidados de enfermagem ao portador de transtorno afetivo bipolar: relato de experiência \\
\hline Estudo 13 & 2012 & A percepção dos trabalhadores de enfermagem sobre a assistência à saúde mental em Hospital Universitário \\
\hline Estudo 14 & 2012 & Atitudes do profissional de enfermagem em relação ao comportamento suicida: influência da inteligência emocional \\
\hline Estudo 15 & 2011 & Abordagem da equipe de enfermagem ao usuário na emergência em saúde mental em um pronto atendimento \\
\hline Estudo 16 & 2011 & Tentativa de autoextermínio em um Hospital da região leste de Minas Gerais \\
\hline
\end{tabular}

Figura 1 - Distribuição dos estudos segundo ano de publicação e título. Florianópolis, SC, Brasil, 2019

\section{Discussão}

Não existe um conceito oficial de saúde mental, já que a mesma é influenciada pelas diferenças culturais e julgamentos subjetivos. Trata-se de um campo de conhecimento e experiência psíquica e, como tal, é polissêmico. Esta concepção sustenta-se sob três dimensões: a) transversal à saúde - valoriza-se a subjetividade de cada pessoa e família; b) é uma especificidade - a intervenção é junto à pessoa em sofrimento psíquico; c) é uma especialidade promovem-se ações em saúde com pessoas com transtornos mentais severos e graves ${ }^{(8)}$. Saúde Mental "é uma experiência subjetiva e objetiva em essência instável, caracterizada pela capacidade de lidar com as crises, enriquecer a subjetividade e lidar com a própria experiência psíquica"(9).
A Política Nacional de Saúde Mental redireciona o modelo assistencial em saúde mental, preconizando uma rede de serviços e equipamentos sociais variados, tais como os Centros de Atenção Psicossocial (CAPS), os Serviços Residenciais Terapêuticos (SRT), os Centros de Convivência e Cultura e os leitos de atenção integral (em hospitais gerais, nos CAPS III). Esses espaços acolhem pessoas com transtornos mentais como também usuários de álcool e outras drogas, modificando a estrutura da assistência à saúde mental(10).

Esses dispositivos vêm substituindo progressivamente o modelo hospitalocêntrico e manicomial, de características excludentes, opressivas e reducionistas na tentativa de construir um sistema de assistência orientado pelos princípios fundamentais do SUS - universalidade, equidade e integralidade. Este tipo de atendimento é resultado de um longo processo 
de luta social que culminou com a Reforma Psiquiátrica, em 2001, cuja principal bandeira está na mudança do modelo de tratamento: no lugar do isolamento, o convívio com a família e a comunidade ${ }^{(11)}$.

A família e os equipamentos sociais são indispensáveis para a promoção de uma melhor qualidade de vida. A Psiquiatria, a loucura e o destino dos pacientes psiquiátricos são questões debatidas pela sociedade e deixaram de ser um problema restrito aos especialistas, e cabe a todos os tipos de instituições o cuidado desse paciente, não se restringindo apenas aos locais especializados ${ }^{(12-13)}$.

\section{Contextualização do tema suicídio no âmbito da urgência e emergência}

Em uma perspectiva histórica, o ato de acabar com a própria vida está rodeado pelo tabu e pelo preconceito, gerando um efeito desconfortável entre as pessoas. A abordagem à pessoa com transtorno mental em situação de emergência, quando realizada com disposição, segurança, prontidão e qualidade, é capazes de determinar a aceitação e a adesão dessa pessoa ao

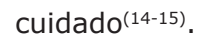

A tentativa de autoextermínio refere-se a atos altamente letais que não resultam em morte, porém, apresenta consequências como, transtornos para os familiares, gastos elevados no setor público, procedimentos invasivos e ocasiona consequências graves na saúde desse e dessa paciente. Isso tem sido um problema frequente nos serviços hospitalares de urgência, necessitando de avaliação e cuidados que visam à redução dos danos autoinfligidos ${ }^{(16-17)}$.

A pessoa em risco de suicídio requer medidas adequadas de ambientação, sendo preciso desconstruir o princípio da periculosidade e da incapacidade, onde a pessoa é infantilizada - característica da assistência psiquiátrica asilar e do senso comum - e promover o convívio, estimulando a inclusão e a autonomia, características da atenção psicossocial. A inserção da pessoa com transtorno mental nas unidades de internação hospitalares e o aumento do número de atendimentos a essa demanda, exigem que o hospital ofereça assistência qualificada, incluindo a capacitação da equipe assistencial para o atendimento em saúde mental, a disponibilidade de recursos de materiais e de equipe, e a elaboração de protocolos de atendimento $^{(12,18)}$.

Segundo os autores dos estudos 4 e 8 , os fatores que são predisponentes para uma pessoa tentar suicídio são aqueles que podem criar condições favoráveis, como idade, gênero e a existência prévia de agravos à saúde. Esses fatores podem ser elencados como: facilitadores, desencadeantes e potencializadores. Os fatores facilitadores podem atuar, contribuindo para a ocorrência, como alimentação inadequada ou condições habitacionais desfavoráveis ao evento. Os fatores desencadeantes podem associar-se ao aparecimento da intoxicação ou evento adverso à saúde, como a exposição a determinados agentes. Os fatores potencializadores podem agravar uma intoxicação já estabelecida, como, por exemplo, a exposição repetida ou por tempo prolongado a condições adversas ${ }^{(13,19)}$.

Diversas são as circunstâncias que podem levar ao risco de suicídio em ambos os sexos. Por serem produtoras de estresse, podem-se destacar o estado depressivo, a tentativa anterior de suicídio, o uso de drogas ilícitas, desemprego, aposentadoria, estar em licença médica, pobreza, perda de uma pessoa querida, desentendimentos com familiares ou amigos, término de uma relação afetiva, problemas legais ou de trabalho(13,16,20-22).

A pessoa vítima de tentativa de autoextermínio deve ser tratada de maneira integral na sua recuperação clínica e psíquica. Assim, o profissional de Enfermagem deve saber lidar com estas pessoas para diminuir a recidiva desses casos no atendimento de urgência/ emergência. Cabe ao enfermeiro estabelecer medidas junto à equipe e rotinas que amenizem o preconceito em relação aos pacientes que tentaram tirar a própria vida e viabilizar o convívio social dos mesmos ${ }^{(13,15,17,20,23)}$.

Os dados dos estudos 9 e 13 revelaram que o número de tentativas de suicídio supera o número de suicídios em pelo menos, dez vezes. Apontaram ainda que 15 a $25 \%$ das pessoas que tentam o suicídio voltarão a fazê-lo no ano seguinte e, destes, e $10 \%$ conseguem, efetivamente, matar-se nos próximos dez anos. Em mulheres, esse comportamento acompanha uma tendência mundial. Embora com taxas de suicídio mais baixas que a dos homens, elas apresentam elevadas taxas de tentativa de suicídio, em uma frequência três vezes superior à dos homens. Os estudos 1 e 2 indicaram que, em geral, os homens buscam métodos violentos e letais, como enforcamento e uso de arma de fogo, enquanto as mulheres optam por métodos menos violentos, como a intoxicação(16-17,19).

Os homens desempenham comportamento pessoal e social que predispõe ao suicídio, tal como a competitividade, a impulsividade e o maior acesso às tecnologias letais, sendo ainda mais sensíveis às instabilidades econômicas, como nos casos de desemprego e empobrecimento. O alto número de casos evoluídos para a estabilidade do cuidado indica que as mulheres geralmente não desejam o suicídio no sentido de destruição, aniquilamento, mas 
como fuga, esquecimento, escapatória de sua vida presente ${ }^{(17,19-20,24-25)}$.

Nos casos de suicídio, os problemas causados pelo alcoolismo revelam que as habilidades de enfrentamento do dependente podem estar comprometidas, o que pode aumentar o risco de suicídio. Identificou-se que, quando o alcoolismo e a depressão coexistem, os riscos de tentativa de suicídio aumentam, e são apontados como um dos efeitos de curto prazo decorrentes do consumo de álcool, apesar de sua notificação como causa externa, depressão e insegurança foram as características comportamentais mais frequentes. Assim, mesmo em ambiente de urgência e emergência, cabe à Enfermagem assistir a todos os membros da família, auxiliar na compreensão do problema e auxiliar nos mecanismos de enfrentamento, de forma a obter e manter a saúde da família(15,20-23,25).

De um modo geral, os estudos apontaram que os profissionais de urgência/emergência associam a pessoa que tentou suicídio ao paciente psiquiátrico e, dessa forma, fazem uma relação direta ao estigma. Referem-se à pessoa com ideação suicida, como um paciente que apresenta um comportamento desorganizado e agressivo. As atitudes relacionadas ao estigma e ao preconceito podem ter impacto na prática de Enfermagem de uma maneira que desconstrói o "ser enfermeiro", na qual o profissional busca outra atividade e desse modo, esquiva-se de prestar o cuidado ao paciente em tentativa de autoextermínio(13,20,26).

Por vezes, o estigma é considerado pelo profissional como um defeito, uma fraqueza, uma incapacidade e, mesmo com o acolhimento adequado, o paciente pode ainda sentir necessidade de estar entre os seus semelhantes e não reconhecer a emergência como um local apropriado. O que torna a pessoa diferente está além do comportamento desorganizado; o que torna essa pessoa diferente é a forma como aqueles que não apresentam tais alterações o percebem e o enfrentam ${ }^{(15,22)}$.

A inserção da pessoa com transtornos mentais no âmbito hospitalar exige que os profissionais busquem formas de assistir essa pessoa. Os profissionais costumam priorizar os aspectos clínicos do paciente, enquanto os sintomas psíquicos tendem a ser desvalorizados e tratados de forma equivocada, não sendo identificados pela equipe. Portanto, é importante levar em consideração as mudanças do desenvolvimento técnico-científico, como também a Política de Saúde Mental que, por sua vez, repercute em uma nova construção cultural, onde o lugar do "Iouco" não é mais somente no hospital psiquiátrico, mas nos hospitais gerais, inserido nas Equipes de Saúde da Família, no domicílio, e outros segmentos. Integrar ações de saúde mental e de atenção psicossocial em todos os contextos de assistência de Enfermagem de hospitais gerais qualifica e otimiza o cuidado(12,18).

Atitudes estigmatizantes influenciam negativamente a atenção e o tratamento oferecido aos pacientes e, além disso, têm impacto no seu bem-estar, atuando como obstáculo considerável para a busca de ajuda, acesso ao tratamento, adesão e eficácia do mesmo. Os estudos no âmbito de urgência/emergência indicaram, ainda, que existe um preconceito comum entre médicos e profissionais de Enfermagem sobre os pacientes que se autolesionam, descrevendo-os como manipuladores e chamadores de atenção(15-16).

Os profissionais de Enfermagem enfrentam uma quantidade considerável de trabalho emocional na sua prática diária, tais como problemas como a agressão, desconfiança, depressão ou comportamento suicida. Esse trabalho profissional é rodeado por sentimentos e emoções, às vezes difíceis de identificar, que têm suas origens tanto no paciente como no próprio profissional. O conjunto de cargas psíquicas presente no cotidiano da Enfermagem pode afetar a sua qualidade de vida e sua competência. Identificar essas emoções e aprender a gerenciá-las supõem a aquisição de novas ferramentas para realizar esse trabalho com sucesso(15,21,27).

Os estudos 1, 2, 4, 9 e 12 afirmaram que profissionais que refletem melhor aceitação do comportamento suicida têm maior chance de prestar assistência de saúde positiva aos pacientes suicidas. Como parte da sociedade e da cultura, o profissional de Enfermagem possui atitudes e crenças que afetam sua atuação profissional e influenciam os pacientes com comportamento suicida. O estigma atribuído à pessoa com transtorno mental pode reduzir suas chances de estabelecer vínculos com a sociedade e compartilhar de outros ambientes sociais(13,15-16,24-25).

\section{Trabalho da Enfermagem de urgência/emergência frente às pessoas que tentam suicídio}

Emergência em saúde mental refere-se a uma situação em que há alteração do pensamento ou das ações e que demandam de um atendimento complexo e, às vezes, imediato. Essas alterações estão associadas ao risco de morte, como no suicídio, em situações de alterações psíquicas decorrentes do uso abusivo de substâncias psicoativas ou doenças físicas, em que se deve intervir para que haja a diminuição de agravos. Significativa parte dos serviços de urgência e emergência foca a doença e não a pessoa como um todo e suas necessidades ${ }^{(13,15)}$.

A tentativa de autoextermínio faz parte da rotina dos centros de emergência clínica, logo, toda a equipe 
deve ser capacitada para que haja um atendimento qualificado e humanizado. As atitudes culturais influenciam a comunicação e o estilo de resposta do profissional que trabalha com pessoas em crise, o que reforça a importância da educação permanente em saúde mental. É importante o aprendizado da comunicação terapêutica para o estabelecimento de relacionamento terapêutico entre paciente e profissional $(15,17,28)$.

Para os autores do estudo 6, dentre os serviços de saúde, os de emergências são os que apresentam maior dificuldade em estabelecer um ambiente interacional, isto porque ele possui algumas características peculiares, que tendem a torná-lo um espaço, no qual a comunicação é mecanizada e impessoal. O motivo que leva o enfermeiro de uma emergência a tratar de modo distinto o paciente psiquiátrico, e busca encontrar um motivo que justifique esse abandono é questionável. Diante do questionamento, os profissionais referem não estarem aptos a atender o paciente psiquiátrico, e alegam certo despreparo e desconhecimento sobre o assunto, não tendo a abordagem adequada a esses pacientes $^{(13,22)}$.

Dessa forma, constatou-se a diferença na assistência prestada ao paciente psiquiátrico e ao não psiquiátrico (sendo que ambos se encontravam na mesma condição clínica) e essa diferença não se referiu ao cuidar com maior ou menor zelo, e sim, a não prestação de cuidados específicos ao paciente psiquiátrico. Revelam-se o preconceito e o estigma associados às atitudes desses profissionais e a desqualificação do cuidado prestado por eles ${ }^{(13,16,22,29)}$.

O enfermeiro pode sentir-se fragilizado diante da impotência de não efetuar o cuidado integral ao paciente em tentativa de suicídio, por não saber como lidar com o sofrimento mental da pessoa no momento da assistência. As dificuldades em lidar com a diferença e o preconceito do transtorno mental deixam o profissional desconfortável em receber, no setor, o paciente com comportamento suicida(13,21)

Na relação com o paciente, o cuidar exige atenção e dedicação de quem o faz, esse trabalho demanda do pessoal de Enfermagem, investimento afetivo, visando fortalecer esse vínculo, e, é algo que não está prescrito como uma medicação ou procedimento a ser administrado. É comum a presença do sentimento de angústia no profissional, principalmente quando, ao estabelecer o relacionamento interpessoal, ele experimenta a rejeição, o que poderá despertar, no enfermeiro, sentimento de impotência e até mesmo de incapacidade. As dificuldades de relacionamento com pacientes vivenciadas pela equipe de Enfermagem acarretam tensões e sofrimento pelo trabalho. A falta de habilidade para lidar com determinadas situações e o fato de não saber trabalhar suas ações/reações acarretam, ao enfermeiro, situações de estresse e resultados negativos, como o adoecimento(18).

Não há um enfrentamento da situação de cuidar do paciente em tentativa de suicídio, com isso, os profissionais de Enfermagem utilizam um mecanismo de defesa, como a negação, onde há censura da necessidade do cuidado demandado pelo paciente psiquiátrico, impossibilitando a identificação da demanda apresentada. No que tange ao cuidado, devido à falta de qualificação, ou por sua formação ter sido focada no modelo manicomial, é importante que a equipe de Enfermagem receba e desenvolva habilidades e competências psicossociais para oferecer cuidados que deem suporte às necessidades físicas e psíquicas dos pacientes em geral ${ }^{(13,15,20)}$.

Isso porque a Enfermagem é uma profissão que lida com o ser humano, interage com ele e requer o conhecimento de sua natureza física, social, psicológica e espiritual, e, esse conhecimento pode dar-se por meio da Sistematização da Assistência de Enfermagem. Para lidar com o ser humano, é necessário que se compreenda como um todo, inserido num contexto de vida, com uma história, hábitos e costumes ${ }^{(13,18,29)}$.

O cuidado inclui a capacidade para a escuta e o diálogo, além de disponibilidade para perceber o outro, como um sujeito com potencialidade, resgatando sua autonomia e estimulando a cidadania. À medida que os enfermeiros estigmatizam o paciente psiquiátrico, o cuidado prestado não assume sua potência transformadora, não ajuda sequer ao paciente nas suas situações clínicas apresentadas, e tampouco nas questões psíquicas embutidas em sua essência(13,22,29-30).

Não somente os enfermeiros podem sentir-se constrangidos frente à pessoa com transtorno mental, pois, essa sensação pode ser recíproca, em que estes pacientes psiquiátricos também se sentem intimidados quando próximos desses profissionais. Acolher a pessoa com transtorno mental e atender às suas necessidades físicas e mentais se tornam-se um desafio aos profissionais de Enfermagem, principalmente aos que atuam no âmbito da urgência/emergência. A compreensão do transtorno pode permitir, ao enfermeiro, perceber a loucura não da forma negativa como a história a apresentou, ou como a sociedade a estigmatizou, e efetivamente diminuir o espaço entre os que apresentam ou não transtornos mentais(13,15,22).

\section{Particularidades e necessidades no cuidado ao paciente suicida}

O serviço de urgência/emergência é um ambiente dinâmico, onde a equipe de Enfermagem sofre com a 
sobrecarga de trabalho e que exige esforços desses profissionais para tornar este ambiente favorável à comunicação, pois a aliança terapêutica com o paciente é fundamental. Apesar da importância de escutar o paciente, sabe-se que, no modelo biomédico, existem poucos espaços para a escuta dos anseios, tensões e sofrimentos das pessoas. Para que a interação se efetive, há a necessidade de compreender que ela se faz pela troca de experiências e não de modo unidirecional. Igualmente, a observação é essencial para a comunicação não-verbal e algumas das reações apresentadas pelo paciente podem subsidiar dados importantes para o planejamento e desenvolvimento de ações preventivas ou emergenciais pela equipe de Enfermagem ${ }^{(12,17,22)}$.

O aumento do comportamento suicida na atualidade, gera uma demanda aos serviços de saúde, e o enfermeiro, como cuidador de um ser humano fragilizado, precisa compreender o evento suicida como um pedido de ajuda isento de preconceitos quanto à questão. Somente conhecendo os diversos fatores envolvidos na procura pela morte, que o profissional enfermeiro poderá prestar atendimento humanizado ao paciente, colaborando para amenizar o sofrimento psíquico presente nesses casos. Antes de pensar em qualquer tipo de cuidado ou intervenção, o enfermeiro deve escutar e compartilhar com o sujeito em sofrimento(12-13,19).

As estratégias de prevenção do comportamento suicida devem ir além da avaliação do risco físico. Devem-se utilizar de métodos para incentivar as pessoas a buscarem ajuda de profissionais de saúde quando estiverem enfrentando alguns problemas de cunho emocional, problemas relacionados à sua saúde mental ou abuso de substâncias psicoativas. Ressalta-se a importância, em particular, da equipe de Enfermagem, que tem um papel importante no processo de educação em saúde e na vigilância de $\operatorname{casos}^{(18,23)}$.

A base da ação de Enfermagem Psiquiátrica é a comunicação efetiva, competência esta que requer investimento de habilidades teóricas e práticas. Quando o enfermeiro faz uso do vínculo terapêutico como umas das principais ferramentas de trabalho, permite conhecer-se como um instrumento de seu trabalho e também, desse modo, transmitir segurança ao paciente, que passa a se sentir mais seguro e capaz de externar seus conflitos ${ }^{(18,21)}$.

A comunicação é um processo mediado pela compreensão e o compartilhamento de ideias e mensagens transmitidas e recebidas, as quais influenciam o comportamento das pessoas, permitindo que elas expressem suas peculiaridades dentro de um campo interacional. Os profissionais reconhecem que a comunicação se traduz em cuidado e é mediadora da interação entre a equipe de Enfermagem e o paciente com transtorno mental. Desse modo, a comunicação é primordial na efetivação do relacionamento interpessoal, e, é um importante instrumento para o planejamento e o desenvolvimento de tais cuidados, sendo um fenômeno integrador, complexo e humano(18,22,26).

O enfermeiro psiquiátrico é importante aos cuidados complexos às pessoas com transtorno mental. Porém, supõe-se que a escassez desse profissional seja uma razão dessa ausência no discurso, além da interconsulta de Enfermagem Psiquiátrica ser pouco realizada e desconhecida por grande parte dos enfermeiros. Além das questões de qualificação profissional, os estudos apresentaram como potencialidade para a assistência em saúde mental, a presença de profissionais da área "psi", psicólogo e psiquiatra, destacados como possíveis orientadores da assistência à pessoa com transtorno mental. No entanto a participação destes é limitada, afinal nem sempre são reconhecidos no cotidiano assistencial. Com isso a importância do trabalho interdisciplinar que resulta em respostas efetivas e qualificadas, tanto na assistência às necessidades do paciente quanto na capacitação da equipe ${ }^{(18,23)}$.

Outro fator em destaque nos estudos são os riscos que podem surgir ao se implantar a interconsulta de Psiquiatria, pois ela pode contribuir para a divisão de tarefas entre "profissionais da mente e do corpo", influenciando o modelo biomédico e a fragmentação do cuidado. No entanto, a interconsulta é uma tecnologia assistencial capaz de superar a dicotomia físico-psíquica na assistência aos pacientes internados e possibilitar a integralidade da assistência ${ }^{(18,21,26)}$.

Diversos fatores influenciam o cuidado de Enfermagem ao paciente suicida. A principal limitação apontada pelos profissionais de Enfermagem tem sido a fragilidade da sua formação no campo da saúde mental, e a estrutura física das instituições não são apropriadas a receber um paciente dessa especialidade. Os trabalhadores apresentam dificuldades para o cuidado de Enfermagem aos pacientes em sofrimento psíquico. As ações que atualmente inserem esse cuidado nos hospitais gerais ainda carecem de incentivo e, na área de Enfermagem, requerem uma mudança cultural e técnica para o desenvolvimento de ações de atenção psicossocial em todos os contextos de assistência(18,23).

É necessário intervir no processo educativo para que sejam envolvidos os gestores, profissionais e pacientes. E cabe ao enfermeiro buscar, na instituição à qual está vinculado, a capacitação para assistir, com qualidade, essa demanda. A capacitação em saúde mental é uma necessidade, e deve ser desenvolvida tendo-se como referência as mudanças no modelo assistencial 
da Reforma Psiquiátrica e, consequentemente, a inserção da pessoa com transtorno mental no hospital geral. Para muitos trabalhadores, há uma resistência em estabelecer a aproximação com o paciente em tentativa de suicídio, já que existe uma dificuldade em compreender o seu papel de profissional perante essa pessoa. Isso demonstra a necessidade de capacitação, a não aceitação e a falta de habilidade para realizar o cuidado de Enfermagem em saúde mental( $13,15,18)$.

A educação em saúde, a partir do vínculo com o ser humano/paciente e a família, é um aspecto importante para o sucesso da prevenção. Com base em uma visão integral do indivíduo e do conhecimento dos motivos que levam as pessoas a esses atos extremos, o enfermeiro pode colaborar para amenizar o sofrimento presente nas tentativas de suicídio. É necessário investir na saúde mental dos próprios trabalhadores para que se possa construir a assistência integral aos internados ${ }^{(18-19)}$.

A educação permanente nesse âmbito deve incluir o conhecimento sobre as mudanças políticas que vêm ocorrendo nessa área, bem como, a ressalva sobre a transição da prática do cuidado hospitalar, que visava à contenção do comportamento, e hoje para a incorporação de princípios de uma prática interdisciplinar, com o objetivo de conscientizar e qualificar tanto os novos funcionários, quanto os mais experientes sobre o papel do profissional como agente transformador. Assim, a educação permanente, no campo da saúde mental, tem como desafio consolidar a Reforma Psiquiátrica(15,19).

Os serviços de emergência e as instituições de saúde, ao promoverem programas de educação continuada têm como objetivo instrumentalizar os profissionais de Enfermagem para o cuidado aos pacientes com transtorno mental. Desse modo, a temática interação e comunicação deve ser enfatizada devido à sua complexidade e, sobretudo, à sua importância para o processo de cuidar $(16,22,28)$.

\section{Considerações finais}

O estudo buscou evidências científicas para caracterizar o cuidado de Enfermagem prestado às pessoas que tentam suicídio nos serviços de atendimento às urgências e emergências, trazendo subsídios gerais para os profissionais compreenderem alguns conceitos sobre o suicídio, o trabalho da Enfermagem no âmbito da urgência e emergência e as particularidades e necessidades da Enfermagem e do paciente suicida durante $o$ ato de cuidar.

As percepções da equipe de Enfermagem ao cuidar de uma pessoa que tenta suicídio são permeadas de preconceitos e mitos, o que retrata certo despreparo da equipe para lidar com essas situações de estresse.
Enfrentar medos e cuidar do outro é um exercício diário da equipe de Enfermagem que precisa ser enfatizado quando a pessoa cuidada revela problemas de saúde mental, por vezes retratadas pela tentativa de suicídio.

Por fim, cumpre destacar que se trata de tema relevante, com grande necessidade de realização de novos estudos para aprimorar e gerar novas fontes de intervenções junto aos pacientes e aos serviços envolvidos, buscando medidas preventivas que melhorem a qualidade da assistência prestada.

Percebe-se a ausência de mais estudos na área do cuidado e dos métodos que otimizem a assistência de Enfermagem para o paciente que tentou suicídio; assim, recomenda-se a expansão de estudos nessa área de conhecimento da Enfermagem.

\section{Referências}

1.Botega JN, Rapeli CB,Cais CFS. Comportamento Suicida. In: Botega NJ, organizador. Prática psiquiátrica no hospital geral: interconsulta e emergência. Porto Alegre: Artmed; 2012. p. 431-45.

2.Ferreira CLB. Trajetória do acompanhamento em saúde mental dos pacientes após o atendimento na unidade de emergência por tentativa de suicídio [dissertação]. Florianópolis: Universidade Federal de Santa Catarina, Residência Multiprofissional em Saúde; 2014.

3.Vidal CEL,Gontijo ED. Suicide attempts and hosting in emergency services: the perception of those who try. Cad Saude Colet. 2013;2(21):108-14. doi: 10.1590/ S1414-462X2013000200002.

4.Botega NJ. Suicidal behavior: epidemiology. Psicol USP. 2014;25(3):231-36.doi:10.1590/0103-6564D20140004. 5.Teixeira E, Medeiros HP, Nascimento MHM, Silva BAC, Rodrigues C. Integrative literature review step-by-step \& convergences with other methods of review. Rev Enferm UFPI. 2013; 2Spe:3-7.doi: 10.26694/reufpi.v2i5.1457.

6.Ganong $\mathrm{LH}$. Integrative reviews of nursing research. Res Nurs Health.1987 Fev;10(1):1-11.doi: 10.1002/ nur.4770100103.

7. Bardin L. Análise de conteúdo. São Paulo: Edições 70; 2004.

8.Esperidião E, Silva NS, Caixeta CC, Rodrigues J. The Psychiatric Nursing, ABEn and the Scientific Department of Psychiatric and Mental Health Nursing: progress and challenges. Ver Bras Enferm. 2013;66 Spe:171-6. doi: 10.1590/S0034-71672013000700022.

9.Organización Panamericana de la Salud. Salud Mental Guía del Promotor Comunitario [Internet]. 2009 [Acceso 20 oct 2016]. Estrategias para la promoción de la salud mental. Disponible en: http://iris.paho.org/xmlui/ bitstream/handle/123456789/31342/salud-mental-guiapromotor. pdf? sequence $=1$ \&isAllowed $=y$ 
10. Ministério da Saúde (BR). Portal da Saúd. Conte com a Gente. [Internet]. Brasília: Ministério da Saúde; 2013. [Acesso 8 jun 2015]. Disponível em: http://portalsaude. saude.gov.br/index.php/cidadao/acoes-e-programas/ conte-com-a-gente

11. PensesuS - A reflexão fortalece essa conquista [Internet]. Rio de Janeiro: Fundação Oswaldo Cruz; 2014 . [Acesso 8 jun 2015]. Disponível em: http:// pensesus.fiocruz.br/saude-mental

12. Estrela KSR, Loyola CMD. Administration of medication to use when needed and the care of psychiatric nursing. Rev Bras Enferm. 2014 Aug;67(4):563-67. doi: 10.1590/0034-7167.2014670410.

13. Elias ADS, Tavares CMM, Cortez EA. Impact of stigma of madness on the attention of nursing to psychiatric patient in emergency. Cienc Cuid Saúde. 2013 OctDec;12(4):776-83. doi: 10.4025/cienccuidsaude. v12i4. 22553.

14. Caveião C, Hey AP, Montezeli JH, Sales WB, Visentin $A$, Kaled M. Mental patients in an emergency: call difficulties perceived by nursing staff in a mixed unit. Cad Esc Saúde. 2015 [cited 2019 July 12];2(14):21-31. Available from: http://portaldeperiodicos.unibrasil.com. br/index.php/cadernossaude/article/view/2439

15. Kondo EH, Vilella JC, Borba LO, Paes MR, Maftum MA. A nursing team's approach to users ofa mental health emergency room. Rev Esc Enferm USP. 2011 Apr; 45(2):501-7. doi: 10.1590/S0080-62342011000200028. 16. Fernandes MA, Sousa LEN, Sousa AR, Evangelista MF. Nursing care for patients with bipolar affective disorder: a report of experience. Rev Enferm UFPI. [Internet]. 2012 [cited 2019 July 12];2(1):135-8. Available from: https://ojs.ufpi.br/index.php/reufpi/ article/download/727/pdf

17. Souza CRF, Ribeiro DCSI, Vieira MS, Erthal LML. Tentativa de auto-extermínio em um hospital da região leste de Minas Gerais. Ver Enferm Integr. [Internet]. 2011 cited 2019 July 12];4(1):708-15. Available from: https://www.unileste.edu.br/enfermagemintegrada/ artigo/v4/07-tentativa-de-auto-exterminio-em-umhospital-da-regiao-leste-de-minas-gerais.pdf

18. Silva NG, Silva PP, Oliveira AGB. A percepção dos trabalhadores de enfermagem sobre a assistência à saúde mental em hospital universitário. Cienc Cuid Saude. 2012 abr-jun;11(2):302-10. doi: 10.4025/ cienccuidsaude.v11i2.11181.

19. Trevisan EPT, Santos JAT, Oliveira MLF. Suicide attempts in women: data from a toxicological assistance center in Parana. Reme Rev Min Enferm. 2013;17(2):412-7. doi: 10.5935/1415-2762.20130031. 20. Silva JCS, Coelho MJ, Cavalcanti ACD, Pinto CMI, Santos MSS, Lima EMS. Poisoned men as subjects of care and nursing care. Esc Anna Nery. 2014 Dec;18(4):71621. doi: 10.5935/1414-8145.20140102.

21. Mangueira SO, Fernandes AFC, Pinheiro AKB, Lopes MVO. Clinical indicators of the dysfunctional family processes nursing diagnosis in alcoholics: an integrative review. Rev Eletr Enferm. 2013 Jul/Sep;15(3):819-28. doi: 10.5216/ree.v15i3.17766.

22. Paes MR, Maftum MA. Communication between nursing team and patients with mental disorder in an emergency service. Cienc Cuid Saúde. 2013;12(1):5562. doi: 10.4025/cienccuidsaude.v12i1.15830.

23. Reis LM, Martins BF, Gavioli A, Mathias TAF, Oliveira MLF. Men's health: hospital admissions for poisoning recorded in a toxicology treatment center. Esc Anna Nery. 2013Aug.;17(3):505-11. doi: 10.1590/ S1414-81452013000300014.

24. Tavares CMM, Cortez EA, Muniz MP. Care in psychiatric hospital under the perspective of a nursing team. Rev RENE. 2014 Jun;15(2):282-90. doi: 10.15253/2175-6783.2014000200013.

25. Carmona-Navarro MC, Pichardo-Martínez MC. Attitudes of nursing professionals towards suicidal behavior: influence of emotional intelligence. Rev. Latino-Am. Enfermagem. 2012 Dec; 20(6):1161-8. doi: 10.1590/S0104-11692012000600019

26. Bahia CA, Avanci JQ, Pinto LW, Minayo MCS. Selfharm throughout all life cycles: profile of victims using urgent and emergency care services in Brazilian state capitals. CienSaude Col. 2017;22(9):2841-50. doi: 10.1590/1413-81232017229.12242017.

27. Brixner $B$, Koch $C L$, Marth MP, Freitas AP, Garske CCD, Giehl VM, et al. Methods used in suicide attempts and sociodemographic characteristics of patients treated at the emergency department of a teaching hospital. Sci Med. 2016;26(4):ID24467.doi: 10.15448/1980-6108.2016.4.24467

28. Gonçalves PIE, Silva RA, Ferreira LA. Comportamento suicida: percepções e práticas de cuidado. Psicol Hosp. [Internet]. 2015;13(2):64-87. [citado em 19 jul 2019]. Disponível em: http://pepsic.bvsalud.org/pdf/ph/ v13n2/13n2a05.pdf

29. Martins GCS, Peres MAA, Oliveira AMB, Stipp MAC, Almeida AJ Filho. The stigma of mental illnesses and the therapeutic residences in the town of Volta Redonda-RJ. Texto Contexto Enferm. 2013 Jun; 22(2):327-34. doi: 10.1590/ S0104-07072013000200008.

30. Silva SL, Kohlrausch ER. Pre-hospital care to the individual with suicidal behavior: an integrative review. SMAD, Rev Eletrônica Saúde Mental Alcool Drog. 2016 Apr-Jun;12(2):108-15. doi: 10.11606/issn.1806-6976. v12i2p108-115 


\section{Contribuição dos autores}

Concepção e planejamento do estudo: Mayara Cristine Fontão, Jeferson Rodrigues, Monica Motta Lino e Murielk Motta Lino. Obtenção dos dados: Mayara Cristine Fontão, Jeferson Rodrigues, Monica Motta Lino e Murielk Motta Lino. Análise e interpretação dos dados: Mayara Cristine Fontão, Jeferson Rodrigues, Monica Motta Lino e Murielk Motta Lino. Análise estatística: Mayara Cristine Fontão, Jeferson Rodrigues, Monica Motta Lino e Murielk Motta Lino. Redação do manuscrito: Mayara Cristine Fontão, Jeferson Rodrigues, Monica Motta Lino e Murielk Motta Lino. Revisão crítica do manuscrito: Mayara Cristine Fontão, Jeferson Rodrigues, Monica Motta Lino e Murielk Motta Lino.

Todos os autores aprovaram a versão final do texto.

Conflito de interesse: os autores declararam que não há conflito de interesse. 